\title{
Energy Storage Management with Bidirectional Energy Control in Residential Application
}

\author{
Geethamahalakshmi $\mathrm{G}^{1}$, Nageswari $\mathrm{D}^{3}$, \\ ${ }^{1,3}$ Assistant Professor, \\ Department of Electrical and Electronics Engineering, \\ R M K College of Engineering and Technology, \\ Thiruvallur, India
}

\author{
Dr. Kalaiarasi $\mathrm{N}^{2}$ \\ ${ }^{2}$ Professor, \\ Department of Electrical and Electronics Engineering \\ R M K College of Engineering and Technology, \\ Thiruvallur, India
}

\author{
Lokeshwaran $\mathrm{R}^{4}$, Ajithdurai $\mathrm{S}^{5}$, Annamalai $\mathrm{N}^{6}$, Gopikannan $\mathrm{K} \mathrm{M}^{7}$ \\ ${ }^{4,5,6,7}$ Student, \\ Department of Electrical and Electronics Engineering, \\ R M K College of Engineering and Technology, \\ Thiruvallur, India
}

\begin{abstract}
The off grid energy production system is widely used in residential renewable application for uninterrupted power usage. This paper is for the bidirectional gird system. However, due to inefficiency over energy storage systems and dynamic and static pricing over the selling and buying of the energy makes the system complex and sophisticated. In this regard, the use of optimizing algorithm in a real time conditions through a micro controller gives a feasible closed loop solution with minimum complexity. This system also gives better structure to energy flow and storage system decision. This system also gives improved utilization of the energy from the renewable energy generation. Simulation helps to show the buying side and selling side voltage, current, harmonics, effectiveness of control strategy and to understand system cost and buying and selling price of energy through the system.
\end{abstract}

Index Terms-Storage system, renewable energy generation, bidirectional grid system, closed loop control, energy flow

\section{INTRODUCTION}

Hybrid grid is considered as the key solution to residential distribution grid. The hybrid is considered to be the future power structure in residential application and this will meet the energy production and distribution in residential renewable production. The local renewable energy production in residential application has a greater flexibility in energy control. The hybrid grid has an ability to buy energy from the conventional grid and to sell the energy produced from the renewable source back to the conventional this makes the system closed loop and bidirectional flow between conventional grid and energy storage systems. This makes the consumers to buy and sell energy through a dynamic or a static pricing scheme.

The grid system requires a better control strategies and effective storage system. The bidirectional flow between the grid and storage unit makes the system complicated and creates more challenges which requires intelligent control strategies more control decision has to be made. A smart control system solution to the total grid creates a better energy flow in real time application this solution helps to maximize the benefits from the system. Moreover, the control strategy has to know about storage unit investment, energy flow between load, conventional grid, renewable grid and storage unit, and has to decide how much of energy to buy, sell, store. The storage unit which is the battery unit has limitations since the battery has to charge and discharge continuously the battery unit requires a better and optimized usage of it.

In this paper proposes a control strategy and energy flow. The proposed system is simulated and low scale prototype is developed and evaluated under various conditions and results show the effectiveness of the system.

\section{A. Related Works}

The Energy Storage Management has been considered at the power grid to overcome the oscillating of renewable generation, with many works in literature on storage control and valuation of its role in renewable generation [1], for power stabilizing with fixed load [2], [3] or flexible load control [5], [6], and for phase stabilizing [4]. Residential energy storage systems to shorten electricity cost have been considered without renewable energy [17] and with renewable integration [9]- [12], [18]- [24]. where combined strategies of load prediction and day-ahead scheduling on respective large and small timescales are proposed. The knowledge of load statistics and renewable generation are known ahead of time, while no battery operational cost are considered. Real-time energy storage management amid unfamiliar system dynamics is much more challenging. Assuming known distributions of system dynamics and solved numerically using Dynamic Programming [13], [18]. However, this method suffers from high computational sophisticated to be implementable for practical systems. In addition, due to unpredictable nature of system dynamics, the required statistics are complicated to acquire or predict in practice. Without the statistical knowledge of system dynamics, for independent and identically distributed or stationary system dynamics (pricing, renewable, and load), energy control algorithms are proposed in [19], [20] without considering battery working cost, and in [21] with battery charging and discharging operational cost considered. All the above works aim to shrink the long-term average 
system cost. A real-time energy control algorithm to minimize the system cost within a finite time period is designed in [11] for arbitrary system dynamics. Furthermore, joint storage control and flexible load scheduling is considered in [12] where the closed-form sequential solution was developed to minimize the system cost while meeting the load deadlines. The idea of energy selling or buying is considered in, where focus on demand-side management via pricing schemes using game approaches for load scheduling among customers, and considers a micro grid operation and supply. In addition, although not explicitly modeled, the system considered in [24] can be generalized to include energy selling under a simplified model, provided that buying and selling prices are constrained such that the overall cost function is still convex. All these works consider the grid level operation and the cost associated with it, and use a simple battery storage model without considering degradation or operational cost. Since the consumers may prefer a cost saving management solution in a customer-defined time period, and system dynamics may not be stationary, it is important to provide a cost-minimizing solution to meet such need. There is no such existing bidirectional energy management solution with energy selling-back capability. In addition, most prevailing works ignore battery inefficiency in charging and discharging, which outcome in energy loss that affects the storage behaviors and should be taken into account in the energy storage control design.

\section{SYSTEM DESCRIPTION}

The system consists of conventional grid which supplies voltage at required frequency, the renewable grid which is photovoltaic energy source or other renewable energy source but for simple application photovoltaic system is used. The conventional grid is connected to the battery, single phase inverter and finally to the load. The photovoltaic source is connected to the charging circuit and then to the battery. The inverter which takes necessary condition to operate the system. Inverter is connected to microcontroller unit which the algorithm is fed and microcontroller gives the control signals to the single phase inverter and other system components.

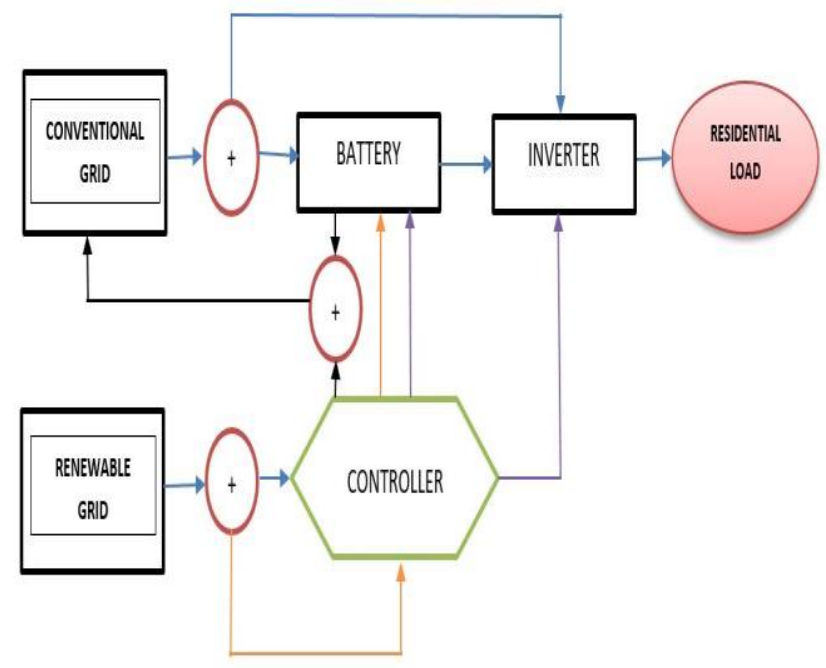

The microcontroller unit which has the condition logic and control is present and takes various input from the conventional grid, photovoltaic source generation, battery state of charge (SOC), other inputs from the inverter output are given to the controller. The microcontroller has the algorithm working under optimized state. The microcontroller is able to identify the demand duration in the conventional grid and makes the decision required to trade the energy in specific time duration.

The microcontroller uses Maximum power point tracking (MPPT) algorithm to maximize the PV source peak power generation. Using coulomb counting method the state of charge (SOC) of batteries are calculated. Inverter also uses maximum power point tracking (MPPT) in simulation PID controller is used to control and evaluation.

\section{OPERATION}

The conventional grid supplies an alternating voltage and frequency of required level the power from the conventional grid is given to the storage unit then to the single phase inverter and to the residential load. Whenever the renewable source does not produce required power from the source the conventional grid charges the battery unit through AC-DC converter.

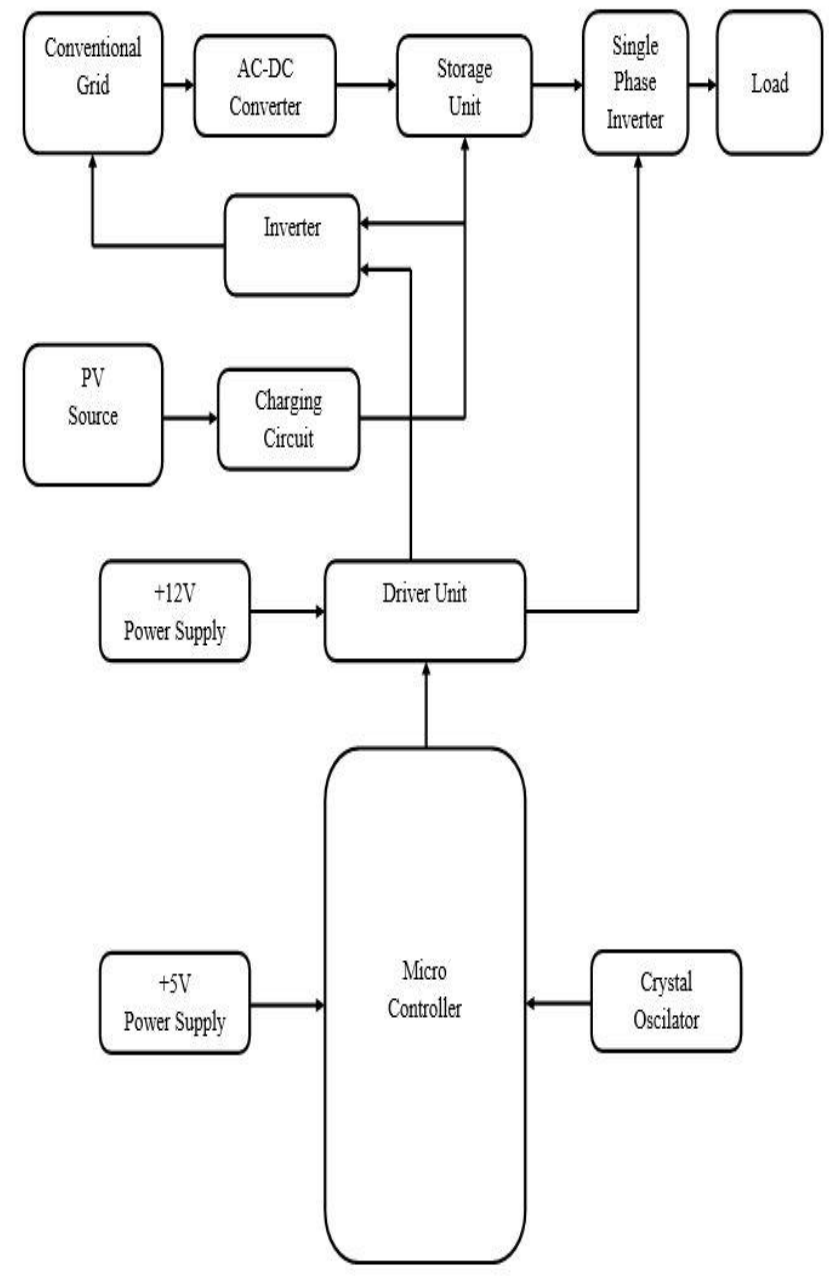

Figure 2. Hardware block diagram

Figure 1. Simple block diagram 
The renewable source generates power and charges the storage unit using charging circuits. During times the charging unit is full during this time the driver unit switch the switches and power from the renewable source if given to the selling side inverter to match the voltage, current and frequency and given to grid.

During demand duration the storage unit charge is discharged up to certain percentage a safe amount of charge is present in storage unit for backup propose. The discharged charge is added with the energy produced from the renewable source. This excess energy gives a suitable amount of power which results in more power traded to the grid and battery life is increased accordingly. The battery lifecycles get reduced accordingly and battery deterioration rate is also calculated by controller and control signal is given.

The microcontroller unit is given with crystal oscillator for signal pluses and connected to high power driver unit which is the switch whenever the microcontroller condition is given to driver unit it takes required action to switch the switches in high power driver unit.

\section{SIMULATION AND EXPERIMENTAL RESULTS}

To verify the system, the proposed system model is simulated in MATLAB software. The design parameters are mentioned in the Table I.

The PV load side has a total of 96 series connected cells and a total of 5 series and 40 parallel modules and at a constant temperature of 25 degrees Celsius and irradiance of 800 .

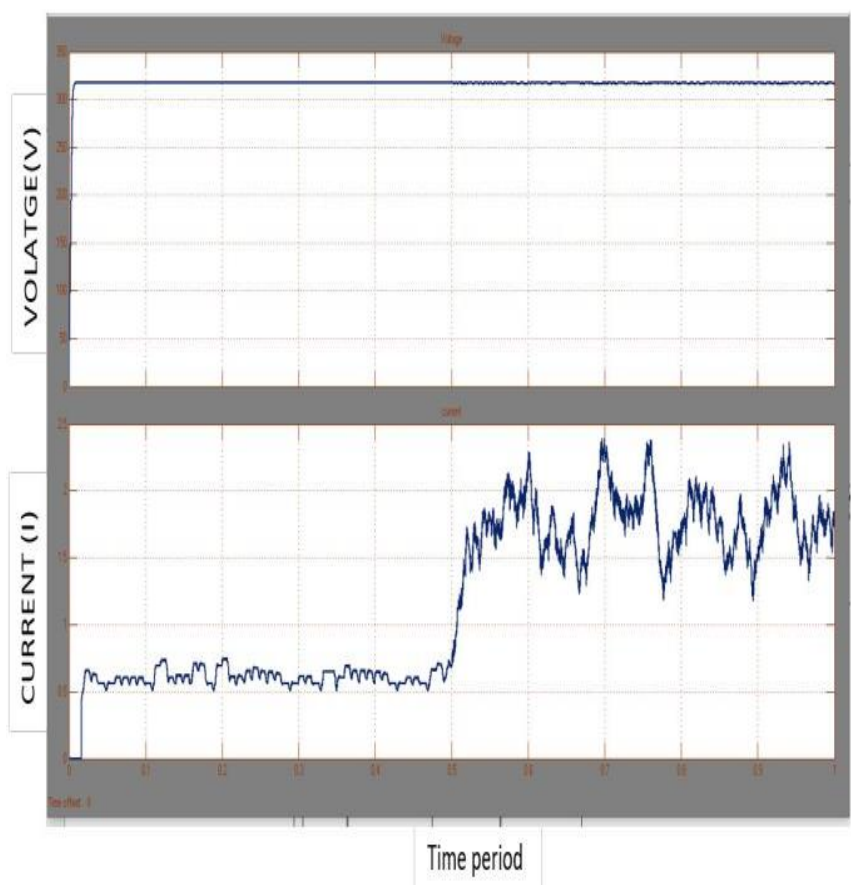

Figure 3. PV panel input voltage and current
Table I: Design parameters

\begin{tabular}{|l|l|l|l|l|}
\hline S.no & Description & Parameter & Value & Unit \\
\hline 1 & $\begin{array}{l}\text { Base voltage in } \\
\text { conventional grid }\end{array}$ & V0 & 230 & $\mathrm{~V}$ \\
\hline 2 & $\begin{array}{l}\text { Base frequency in } \\
\text { conventional grid }\end{array}$ & $\mathrm{f0}$ & 50 & $\mathrm{~Hz}$ \\
\hline 3 & $\begin{array}{l}\text { Nominal battery } \\
\text { voltage }\end{array}$ & Vbat & 400 & $\mathrm{~V}$ \\
\hline 4 & $\begin{array}{l}\text { Nominal battery } \\
\text { capacity }\end{array}$ & Cbat & 10 & $\mathrm{Ah}$ \\
\hline 5 & Load Resistance & RL & 100 & $\Omega$ \\
\hline 6 & Battery Technology & & $\begin{array}{l}\text { Lead- } \\
\text { Acid }\end{array}$ & \\
\hline
\end{tabular}

The output waveform characteristics the waveform gives the voltage of $230 \mathrm{~V}$ at $50 \mathrm{~Hz}$ frequency and at a powerfactor of unity where volatge and current are in phase with each other.

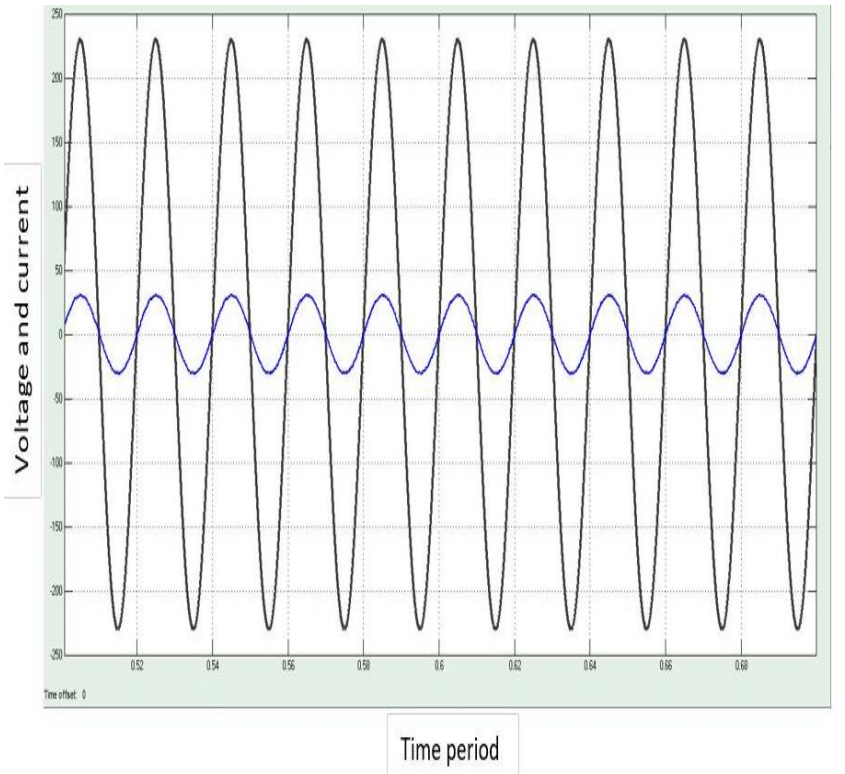

Figure 4. Voltage ( $\mathrm{V}_{\mathrm{op}}$ ) and Current ( $\left.\mathrm{I}_{\mathrm{op}}\right)$ on selling side.

The current given to the load is continous without any interruption during the switching of the inverter the flow of current is mantained. During the discharge of the battery to load or to the grid the current given to the residential load is continous.

The volatge and current from the PV module is dc supply and it is not continous because of the radiation the voltage are produced high at radiation duration. The charge controller using Maximum power point tracking (MPPT) module to boost the charge the battery unit. 


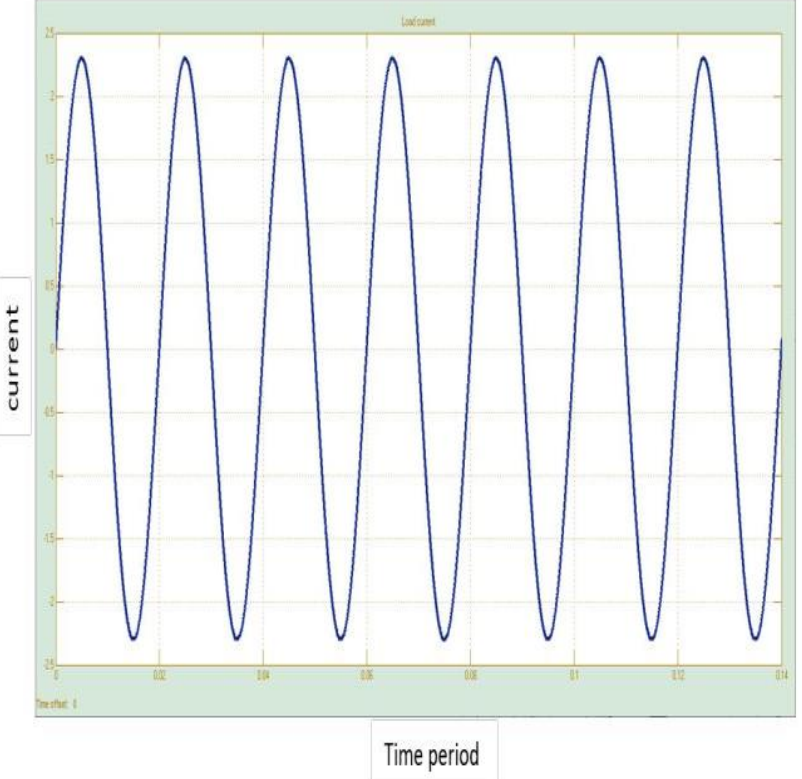

Figure 5. Load Current ( $\left.\mathrm{Irr}_{\mathrm{r}}\right)$ to residential load.

The battery charging and discharging is upon the condition and pluses given by the controller. During power failure the battery discharges and during the selling to the grid when the battery is maintained at the nominal voltage the condition is given to the relay unit and the relay unit switches the direction of flow of charge.

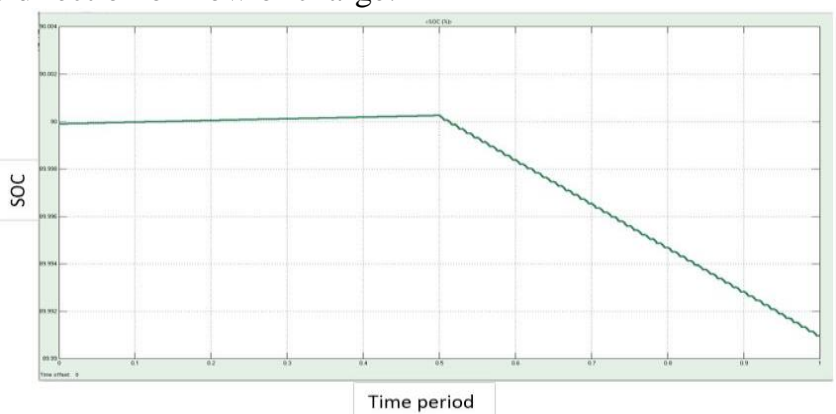

Figure 6. Switching of battery during demand conditions.

Using FFT analysis harmonics is identified in the simulation and harmonics produced in the system is minimal.

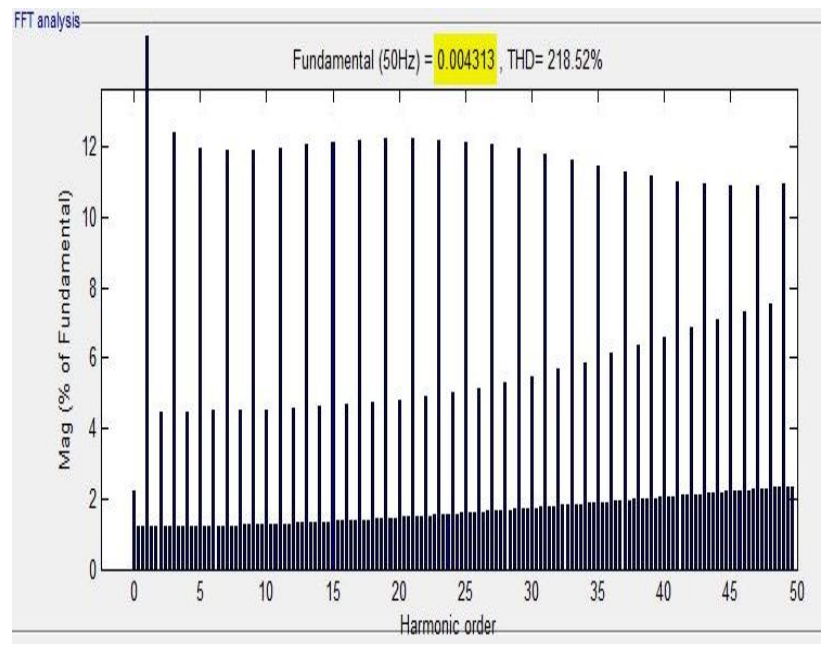

Figure 7. Harmonics.

\section{CONCLUSION}

In this paper we proposed a better hybrid structure in residential application. the renewable source is integrated with the conventional grid to maximize the energy traded to the grid in bidirectional flow a better energy flow through the grid helps to minimize the complexity of the system. The real time control and closed loop control of the system makes easy to be implement and control. This method has better battery storage management. Energy loss during excess energy production in off grid methodologies is subdued using this method of bidirectional flow control. The energy traded is highly beneficial and profitable to the consumer in residential application. Further simulation is used to study and understand the operation of the system and power flow of the system.

\section{REFERENCE}

[1] "Residential Energy Storage Management with Bidirectional Energy Control" Tianyi Li, and Min Dong, Senior Member, IEEE, 2018

[2] P. Denholm, E. Ela, B. Kirby, and M. Milligan, "The role of energy storage with renewable electricity generation," National Renewable Energy Laboratory, Tech. Rep. NREL/TP-6A2-47187, 2010.

[3] "Real-time energy storage management with renewable energy of arbitrary generation dynamics," in Proc. IEEE ASILOMAR, 2013.

[4] H.-I. Su and A. El Gamal, "Modeling and analysis of the role of energy storage for renewable integration: Power balancing," IEEE Trans. Power Syst., vol. 28, pp. 4109-4117, 2013.

[5] S. Sun, M. Dong, and B. Liang, "Real-time power balancing in electric grids with distributed storage," IEEE J. Select. Topics Signal Processing, vol. 8, pp. 1167-1181, 2014.

[6] M. He, S. Murugesan, and J. Zhang, "A multi-timescale scheduling approach for stochastic reliability in smart grids with wind generation and opportunistic demand," IEEE Trans. Smart Grid, vol. 4, pp. 521$529,2013$.

[7] S. Sun, M. Dong, and B. Liang, "Distributed real-time power balancing in renewable-integrated power grids with storage and flexible loads," IEEE Trans. Smart Grid, vol. 7, no. 5, pp. 2337-2349, 2016.

[8] Y. Zhang, N. Gatsis, and G. Giannakis, "Robust energy management for microgrids with high-penetration renewables," IEEE Trans. Sustain. Energy, vol. 4, pp. 944-953, 2013.

[9] A. Faruqui, R. Hledik, and J. Tsoukalis, "The power of dynamic pricing," Electr. J., vol. 22, no. 3, pp. 42-56, 2009.

[10] C. Joe-Wong, S. Sen, S. Ha, and M. Chiang, "Optimized day-ahead pricing for smart grids with device-specific scheduling flexibility," IEEE J. Sel. Areas Commun., vol. 30, pp. 1075-1085, 2012.

[11] Y. Wang, X. Lin, and M. Pedram, "Adaptive control for energy storage systems in households with photovoltaic modules," IEEE Trans. Smart Grid, vol. 5, no. 2, pp. 992-1001, 2014.

[12] S. Sun, B. Liang, M. Dong, and J. A. Taylor, "Phase balancing using energy storage in power grids under uncertainty," IEEE Trans. Power Syst., vol. 31, no. 5, pp. 3891-3903, 2016.

[13] T. Li and M. Dong, "Real-time energy storage management with renewable integration: Finite-time horizon approach," IEEE J. Sel. Areas Commun., vol. 33, no. 12, pp. 2524-2539, 2015.

[14] "Real-time residential-side joint energy storage management and load scheduling with renewable integration," IEEE Trans. Smart Grid, vol. 9, pp. 283-298, 2018.

[15] K. Rahbar, J. Xu, and R. Zhang, "Real-time energy storage management for renewable integration in micro grid: An off-line optimization approach," IEEE Trans. Smart Grid, vol. 6, pp. 124-134, 2015.

[16] M. R. V. Moghadam, R. Zhang, and R. T. B. Ma, "Demand response for contingency management via real-time pricing in smart grids," in Proc. IEEE Int. Conf. Smart Grid Commun. (Smart Grid Comm), 2014, pp. 632-637.

[17] "Grid-connected renewable energy systems," U.S. Department of Energy. "Information for renewable generators," Ontario Energy Board.

[18] J. Qin, Y. Chow, J. Yang, and R. Rajagopal, "Distributed online modified greedy algorithm for networked storage operation under uncertainty," IEEE Trans. Smart Grid, vol. 7, no. 2, pp. 1106-1118, 2016 
[19] Y. Zhang and M. van der Schaar, "Structure-aware stochastic storage management in smart grids," IEEE J. Sel. Topics Signal Process., vol. 8, pp. 1098-1110, 2014.

[20] L. Huang, J. Walrand, and K. Ramchandran, "Optimal demand response with energy storage management," in Proc. IEEE SmartGridComm, 2012.

[21] S. Salinas, M. Li, P. Li, and Y. Fu, "Dynamic energy management for the smart grid with distributed energy resources," IEEE Trans. Smart Grid, vol. 4, pp. 2139-2151, 2013.

[22] T. Li and M. Dong, "Online control for energy storage management with renewable energy integration," in Proc. IEEE ICASSP, May 2013.

[23] A. Castillo and D. F. Gayme, "Grid-scale energy storage applications in renewable energy integration: A survey,” Energy Convers. Manage, vol. 87, pp. 885-894, 2014.

[24] "Real-time energy storage management: Finite-time horizon approach," in Proc. IEEE Int. Conf. Smart Grid Commun. (Smart Grid Comm), 2014, pp. 115-120.

[25] R. Urgaonkar, B. Urgaonkar, M. J. Neely, and A. Sivasubramaniam, "Optimal power cost management using stored energy in data centers," in Proc. ACM SIGMETRICS, 2011. 